\title{
Compatibility of Poly(styrene)/Poly(vinyl acetate) Blends
}

\author{
Heta Rawal and Surekha Devi* \\ Department of Chemistry, Faculty of Science, M.S. University of Baroda, \\ Baroda-390 002, India
}

(Received May 6, 1992)

\begin{abstract}
The blends of poly(styrene)/poly(vinyl acetate) (PS/PVAC) with various compositions were prepared by solution blending technique using methyl ethyl ketone as solvent. The viscosities of the blend solutions were measured and compared with theoretically calculated values. The ternary polymers-solvent systems were found to be miscible. The blends were characterized by FT-IR and thermal studies. The evidence for the interaction between polymeric phases in blends was obtained from FT-IR analysis. The blends under study exhibited improved thermal stability.

KEY WORDS Polymer Blends / Viscosity / Poly(styrene) / Poly(vinyl acetate)

/ Additivity / Interaction / Compatibility /
\end{abstract}

Number of methods have been reported for measuring polymer--polymer compatibility in polymer blends. ${ }^{1}$ Most of them require highly sophisticated instrumentation and a great amount of technical skill. Whereas viscometry is a desirable alternative for studying interaction of polymers in solution. Many researchers ${ }^{2-4}$ have attempted to correlate viscosity with the compatibility for ternary polymer-polymer-solvent systems. It was assumed that repulsion interaction may cause shrinkage of random coils of polymer molecules resulting in reduction in viscosities of the blends when compared with values calculated from the viscosities of the pure components, on the assumption of additivity. On the other hand, in the compatible blends increases in viscosity due to attraction-interaction is expected. In the present study we have attempted to extend this approach for poly(styrene)/poly(vinyl acetate) blend systems.

* To whom correspondences should be addressed.

\section{THEORETICAL BACKGROUND}

For prediction of interaction between binary polymer systems through viscosity behaviour an equation predicting the viscosity behaviour of an ideal system is required. We have considered following two expressions for this purpose. The equation developed by Krigbaum and $\mathrm{Wall}^{5}$ for ideally mixed polymer solutions,

$$
\begin{aligned}
\eta_{\mathrm{sp}(\mathrm{mix})}= & {\left[\eta_{1}\right] C_{1}+\left[\eta_{2}\right] C_{2}+b_{11} C_{1}^{2} } \\
& +b_{22} C_{2}^{2}+2 \sqrt{b_{11} b_{22}} C_{1} C_{2}
\end{aligned}
$$

where $\eta_{\text {sp(mix) }}$ is the specific viscosity of the mixed polymer solution, $\eta_{1}$ and $\eta_{2}$ are intrinsic viscosities of polymer components 1 and 2, respectively. $C_{1}$ and $C_{2}$ are concentrations of components 1 and 2 respectively in the mixed polymer solutions and $b_{11}$ and $b_{22}$ are specific interaction coefficients of components in single polymer solutions. On the other hand a completely empirical equation was developed by Castift and Hewtt. ${ }^{6}$

$$
\eta_{\mathrm{sp}(\mathrm{mix})}=\frac{\left[C_{1}\left(\eta_{\mathrm{sp}(1)}\right)_{c}+C_{2}\left(\eta_{\mathrm{sp}(2)}\right)_{c}\right]}{C}
$$


where $\eta_{\mathrm{sp}(\mathrm{mix})}$ is the specific viscosity of the mixed polymer solution, $\left(\eta_{\mathrm{sp}(1)}\right)_{c}$ and $\left(\eta_{\mathrm{sp}(2)}\right)_{c}$ are specific viscosities of polymer components 1 and 2, respectively at concentration $C$ where $C=C_{1}+C_{2}$, and $C_{1}$ and $C_{2}$ are concentrations of components 1 and 2 , respectively in the blend system. Though this relationship does not have any theoretical justification for definition, ideal behavior of many mixed polymer solutions can be quantitatively predicted using it. In the case of polymers whose solution behavior is described by Huggins equation, ${ }^{7}$

$$
\eta_{\mathrm{sp}} / c=[\eta]+K[\eta]^{2} c
$$

if we put $K[\eta]^{2}=b$, a term which arises from polymer interaction at finite concentration eq 2 may be written as

$$
\begin{aligned}
\eta_{\mathrm{sp}(\mathrm{mix})}= & {\left[\eta_{1}\right] C_{1}+\left[\eta_{2}\right] C_{2}+b_{11} C_{1}^{2} } \\
& +b_{22} C_{2}^{2}+\left(\frac{b_{11}+b_{22}}{2}\right) C_{1} C_{2}
\end{aligned}
$$

The eq 4 is identical to eq 1 except for the coefficient of the $C_{1} C_{2}$ term, i.e., polymer-polymer interaction. For the convenience of computation Krigbaum and Wall defined the specific interaction coefficients for the ideal behaviour of mixed polymer solutions as

$$
b_{12}=\sqrt{b_{11} b_{22}}
$$

However, from eq 4 the interaction coefficient now becomes

$$
b_{12}=\frac{\left(b_{11}+b_{22}\right)}{2}
$$

Occasionally when the value of $b_{11}$ or $b_{22}$ is negative the value of $b_{12}$ according to eq 5 is imaginary. Thus the Krigbaum's definition of $b_{12}$ is unrealistic under these condition. Therefore we have used eq 6 which is a modified form of Castift and Hewtt's defination of ideal behaviour, throughout our work inspite of its empirical base.

Further according to Krigbaum and Wall ${ }^{5}$ information of the interaction between polymer 1 and 2 should be obtained from comparison of experimental $b_{12}$ and theoretical $b_{12}^{*}$ values. Here $b_{12}^{*}$ is referred as the coefficient of interaction between polymer 1 and 2 as computed from eq 6 . The compatibility of polymer mixture is predicted by a parameter

$$
\Delta b=b_{12}-b_{12}^{*}
$$

Negative values of $\Delta b$ are for blend solutions containing incompatible polymers while positive values of $\Delta b$ refer to compatibility of polymers due to attraction interaction.

An alternative defination for ideal solutions may be obtained by considering the intrinsic viscosities of mix polymer solutions

$$
\begin{aligned}
& {\left[\eta_{\mathrm{sp}(\mathrm{mix})} / c\right]_{c \rightarrow 0}} \\
& \quad=\left[\eta_{1}\right]\left[\frac{C_{1}}{C}\right]_{c \rightarrow 0}+\left[\eta_{2}\right]\left[\frac{C_{2}}{C}\right]_{c \rightarrow 0}
\end{aligned}
$$

The results obtained by this process are independent of the value of $b_{12}$ taken from Krigbaum and Wall or from Castiff and Hewtt eq 1 and 4.

Hence eq 2, 7, and 8 can be used to predict the compatibility of poly(styrene)/poly(vinyl acetate), PS/PVA blends.

In the present study the attempts are made to correlate the compatibility of the PS/PVAC blend systems with their viscosities. Further the effect of composition of blends on the compatibility of the system is also studied and blends are characterized through Fourier transfer infrared (FT-IR) and thermogravimetric, (TG) analysis.

\section{EXPERIMENTAL}

\section{Materials}

Poly(styrene) (isotactic, $\bar{M}_{n}=1.3 \times 10^{5}$ ) and poly(vinyl acetate) $\left(\bar{M}_{n}=1.6 \times 10^{5}\right)$ were supplied by Polychem Ltd. Baroda and B.D.H. Chemicals Ltd., U.K., respectively. Poly(styrene) was purified by reprecipitating it from toluene using methanol as nonsolvent. Poly(vinyl acetate) was purified by using chloroform as solvent and ethanol as non solvent. 


\section{Preparation of blends}

Poly(styrene) and poly(vinyl acetate) were dissolved in methyl ethyl ketone (MEK) separately. The solution of the latter was added to the former with constant stirring. Stirring was continued for 3-4 hours. Total concentration of the polymeric mixture in solvent was kept $5 \%$. Films of polymer blends were prepared by casting the solution on the glass plate with an initial slow drying at room temperature. Care was taken to control the uniform thickness of the film for all compositions. To assure complete removal of the solvent final drying was carried out for 24 hours in a vacuum oven at $70^{\circ} \mathrm{C}$ temperature. The compositions of the blends are given in Table I.

The specific viscosities of the polymers and polymer blends were determined by Ubbelohde viscometer. The measurement was carried out in a closed system at $25^{\circ} \mathrm{C}$ to minimize solvent evaporation.

IR spectroscopic information was obtained using Perkin-Elmer Model 1720 Fourier transfer infrared (FT-IR) instrument. Shimadzu-240 spectrophotometer was used to measure the transmittance of the film at $500 \mathrm{~nm}$. Thin polymeric films were examined using Leitz Wetzlar Polarizing Microscope.

\section{RESULTS AND DISCUSSION}

\section{Morphology}

The $\%$ transmittance $(\% \mathrm{~T})$ of the films of the blends at $500 \mathrm{~nm}$ is given in Table II. It is observed that transmittance decreases as the concentration of PS increases. The decrease in transmittance may be due to two reasons. Firstly the crystalline portion of PS forms spherulits and scatters the light and secondly the amorphous region of PS and PVA are not completely miscible and thus form domains acting as scattering centeres. These two factors may act independently or together decreasing $\%$ transmittance.

The optical micrographs for the various solvent cast films of the belnds are illustrated
Table I. Composition of various blends

\begin{tabular}{lcc}
\hline Code no. & $\begin{array}{c}\text { Poly(styrene) } \\
\% \text { (wt/wt) }\end{array}$ & $\begin{array}{c}\text { Poly(vinyl acetate) } \\
\% \text { (wt/wt) }\end{array}$ \\
\hline PS & 100 & - \\
$\mathrm{a}_{1}$ & 90 & 10 \\
$\mathrm{a}_{2}$ & 70 & 30 \\
$\mathrm{a}_{3}$ & 50 & 50 \\
$\mathrm{a}_{4}$ & 30 & 70 \\
$\mathrm{a}_{5}$ & 10 & 90 \\
PVAC & - & 100 \\
\hline
\end{tabular}

Table II. Spectral and thermal properties of polymer systems

\begin{tabular}{|c|c|c|c|c|c|}
\hline \multirow{2}{*}{ Code } & \multicolumn{2}{|c|}{ (TGA) } & \multicolumn{2}{|c|}{ (DTG) } & \multirow{2}{*}{$\% \mathrm{~T}$} \\
\hline & $T_{\max }$ & $/{ }^{\circ} \mathrm{C}$ & $T_{\mathrm{m}}$ & ${ }^{\circ} \mathrm{C}$ & \\
\hline PS & - & 393.0 & - & 416.9 & 27 \\
\hline$a_{1}$ & 326.8 & 404.8 & 346.9 & 425.0 & 30 \\
\hline$a_{2}$ & 320.5 & 405.8 & 432.6 & 428.4 & 32 \\
\hline$a_{3}$ & 323.9 & 408.3 & 343.8 & 433.1 & 35 \\
\hline$a_{4}$ & 322.1 & 413.6 & 342.6 & 429.1 & 60 \\
\hline$a_{5}$ & 322.6 & 423.0 & 344.1 & 426.7 & 72 \\
\hline PVAC & 323.4 & 426.3 & 339.8 & 443.4 & 87 \\
\hline
\end{tabular}

in Figure 1. It can be inferred from the photographs, that with increase in PVAC content, i.e., amorphous phase, the spherulitic organization decreases. The blends at higher PVAC content are nearly amorphous in nature. Since the blends are partially miscible as discussed above, the increase in $\% \mathrm{~T}$ with PVAC content may be probably due to both the factors.

\section{Viscosity Analysis}

Effect of blend composition on intrinsic viscosities is illustrated in Figure 2. The straight line plot of the intrinsic viscosity represents an ideal solution system. The intrinsic viscosity values obtained for present systems were observed to be higher. According to the assumption discussed earlier a positive deviation from ideal solution behavior indicates the force of attraction. Therefore it can be concluded that the polymer systems under 


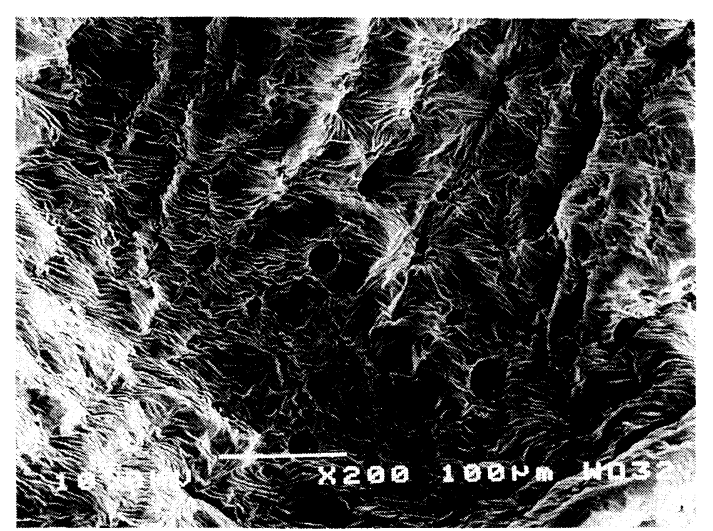

(a)

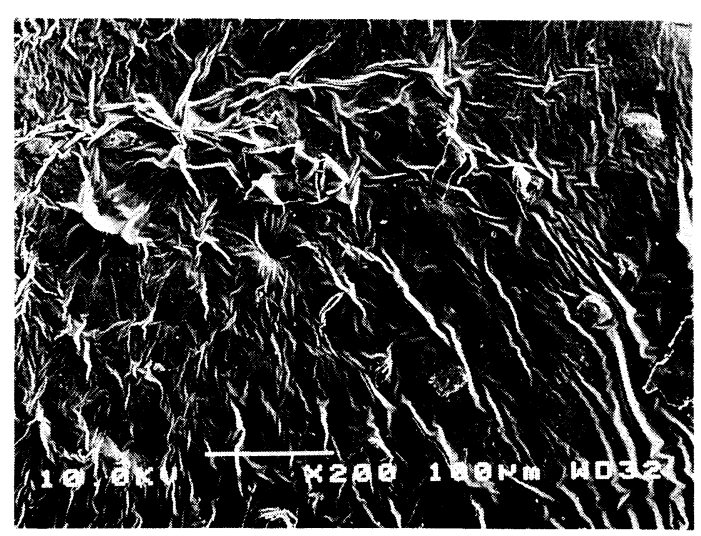

(b)

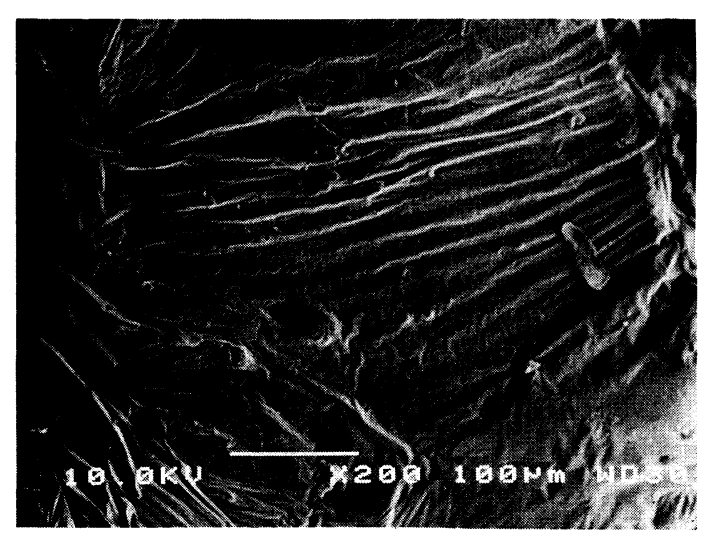

(c)

Figure 1. Optical micrographs of the solvent cast films of PS/PVAC blends: (a), $a_{1} ;$ (b), $a_{3} ;$ (c), $a_{5}$.

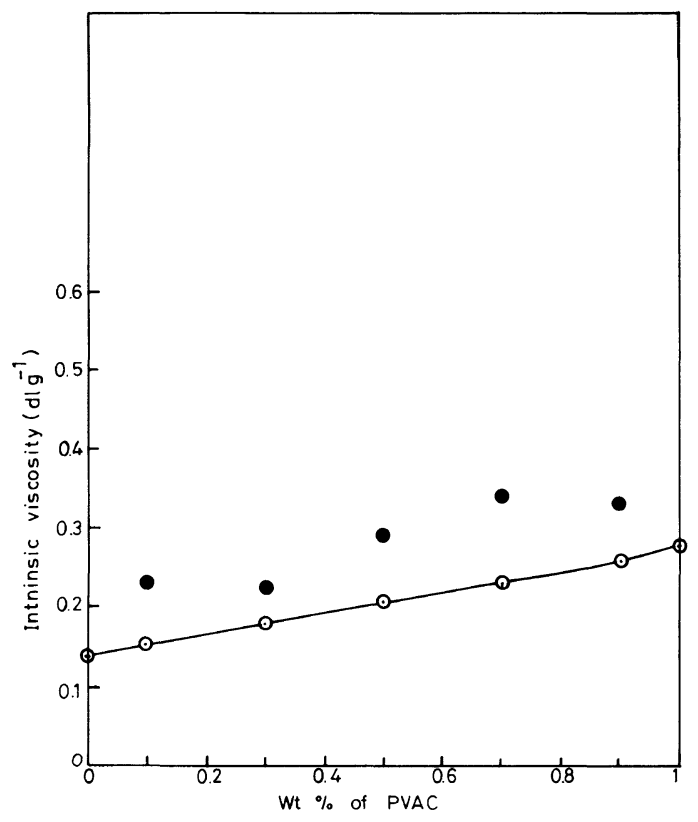

Figure 2. Intrinsic viscosities of PS/PVAC mixture versus composition: $\odot-\odot$. calculated; $-\odot$, experimental.

study may have partially miscible phases. For the blends with $70 \%$ and $10 \%(\mathrm{w} / \mathrm{w})$ PVAC comparatively larger deviation was observed indicating improved miscibility. In Figure 3 the reduced specific viscosities for the various PS/PVAC blends and of homopolymers are presented and are further compared with those of ideally behaving compositions calculated from Castift and Hewtt equation, eq 2. All the blends are found to have more or less positive deviations from ideal values. The $a_{3}$ and $a_{2}$ blends show a little variation at higher concentration from the ideal behavior. However, at lower concentration all the blends are observed to deviate from their ideal behavior. The positive deviation from the ideal behaviour may be interpreted as a sign of polymer interaction. The plot of $\Delta b$ versus concentration of the various blends are shown in Figure 4. The positive value of $\Delta b$ for all the blends indicates some interaction between the phases of the blends. However, with increase in concentration a sharp decrease in the $\Delta b$ values 

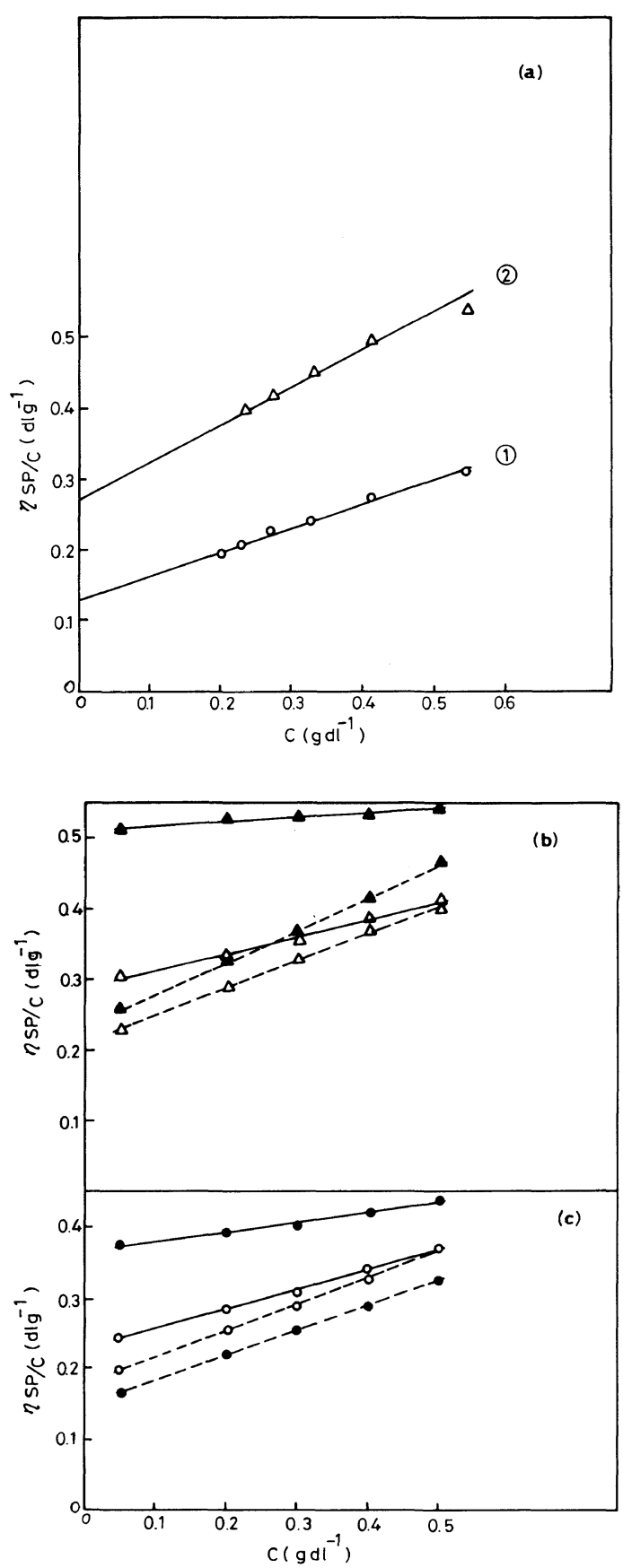

Figure 3. (a) Reduced viscosity versus polymer concentration (1) PS (2) PVAC. (b) Reduced viscosity versus polymer concentration for PS-PVAC-MEK system: $\triangle$, $\mathrm{a}_{3} ; \mathbf{\Delta}, \mathrm{a}_{\mathbf{4}} ;(---)$, theoretical; (-), experimental. (c) Reduced viscosity versus polymer concentration: $\bigcirc, \mathrm{a}_{2} ; \mathbf{O}, \mathrm{a}_{1} ;(---)$, theoretical; (-), experimental.

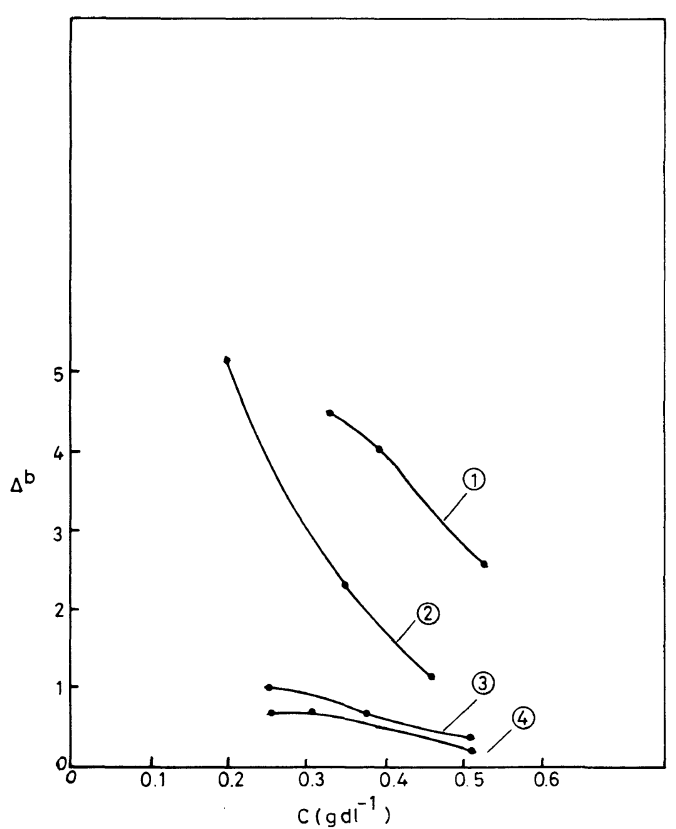

Figure 4. $\Delta b$ versus concentration (1) $\mathrm{a}_{1}$, (2) $\mathrm{a}_{4}$, (3) $\mathrm{a}_{3}$, and (4) $a_{2}$.

is observed, indicating the existence of poor interaction between the phases of the blends.

Thus all the results from viscometric studies in terms of intrinsic, reduced specific viscosities and interaction coefficient $(\Delta b)$ support the presence of some attraction interaction between polymer phases, indicating miscibility of the systems. However, as the interaction is not very strong the system may be only partially miscible.

\section{FT-IR Spectroscopy}

In Figure 5 FT-IR spectra of PS, PVAC, and blends are illustrated. The IR spectra of blends show small shifts in the position of IR bands appearing at $700 \mathrm{~cm}^{-1}$ (for $\mathrm{H}$ out of plane bending of phenyl ring) and a doublet at $1100 \mathrm{~cm}^{-1}$ and $1055 \mathrm{~cm}^{-1}$ (for $-\stackrel{\mathrm{C}}{\mathrm{C}}-\mathrm{O}-$ - stretching). However, this shift was found to vary a little with blend composition. The molecular level mixing occurring in PS/PVAC blends may include dispersion forces from several chemical 
groups. Since bands due to $-\mathrm{CH}$ out of plane show the shift, among many possibilities

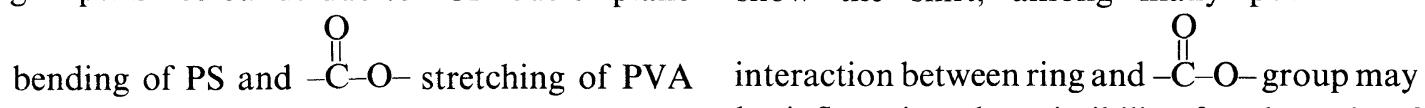
be influencing the miscibility for the pair of

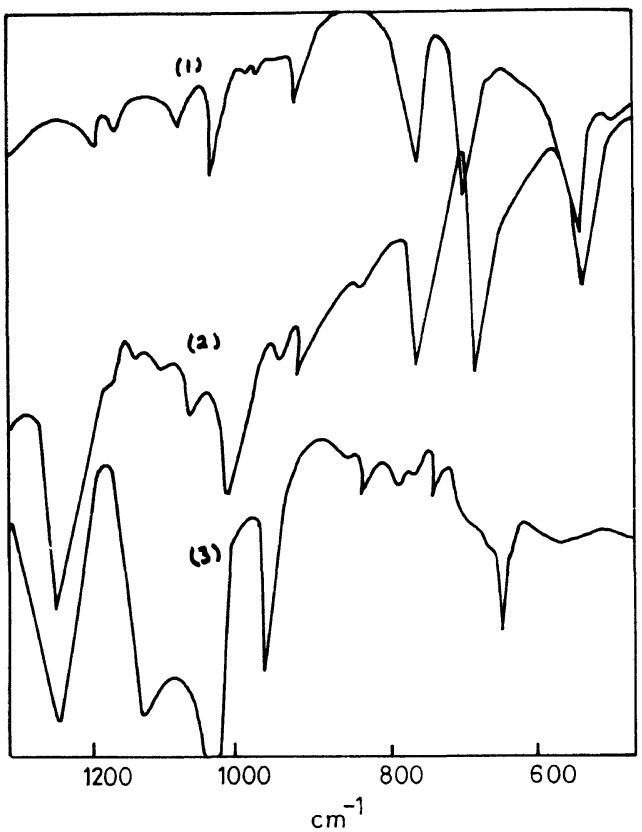
polymers. However, as there is only a small shift in the peak position the interaction between the phases of the polymer may not be very strong indicating partial or poor miscibility. Benedetti et al. ${ }^{8}$ observed $\mathrm{CH}-$ out of plane bending of PS and $\mathrm{CH}_{3} \mathrm{CO}$ stretching more sensitive to change in compatibility of PS/ poly(vinyl methylether) blends.

\section{Thermal Studies}

The TGA results are observed to be providing interesting information. The TGA curves for homopolymers and for blends are shown in Figure 6. The TGA curve for PS shows a sharp transition at $393^{\circ} \mathrm{C}$ due to the decomposition of PS to its dimers and trimers or monomers. PVAC shows two transitions, first at $323^{\circ} \mathrm{C}$ due to degradation of polyene structure. If there is no attraction between two polymers, the TGA curve for the blends is Figure 5. FT-IR spectra of (1) PS, (2) $a_{2}$, and (3) PVAC. expected to lie between those of PS and PVAC.

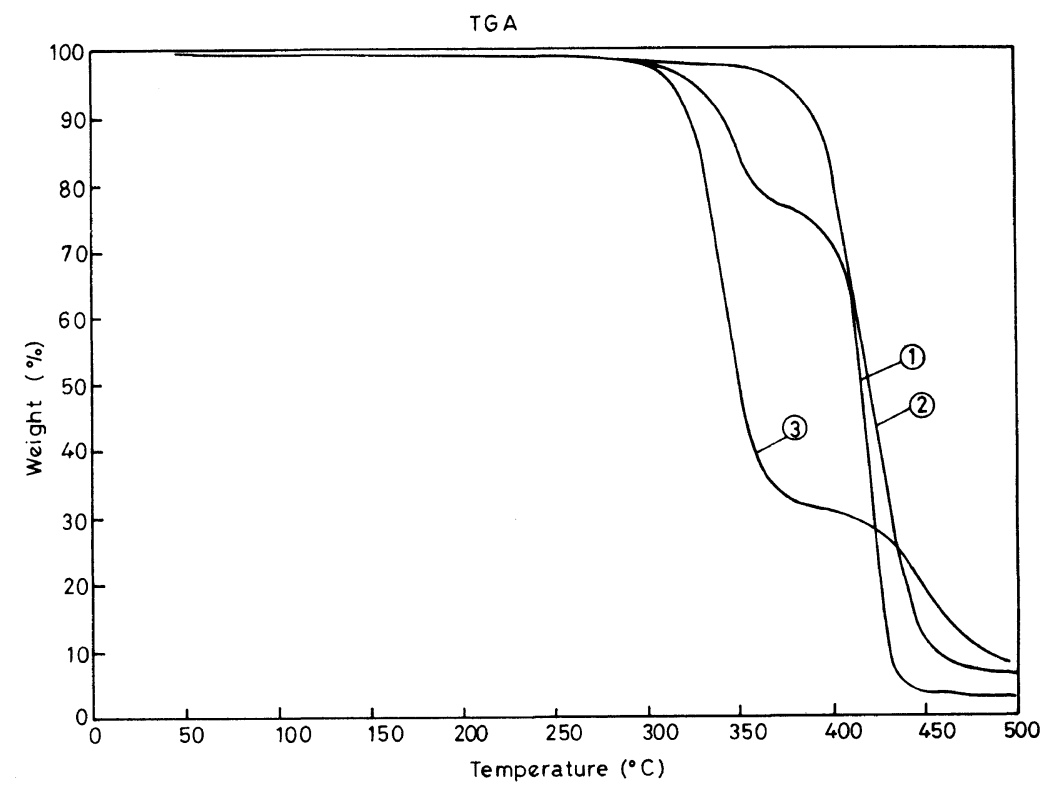

General V4.00 DuPont 2000

Figure 6. TGA thermograms of (1) PS, (2) $\mathrm{a}_{4}$, and (3) PVAC. 
However, it can be seen that at higher temperature the curve is deviated due to improved stability. No significant change is observed in the $T_{\max }$ of PVAC in the blends. However, $T_{\max }$ of PS is shifted to higher temperature. The various $T_{\max }$ values obtained from TGA curves, and DTG (differential thermogravimetric) curves are given in Table II. The higher stability of the blends may be attributed to the interaction of degrading PS with the residue of PVAC deacetylation. From the thermogram it is also observed that the blends show the higher percentage of residue than that of PS confirming the improved thermal stability.

\section{CONCLUSION}

PS/PVAC blends were found to have non ideal value of $\left[\left(\eta_{\mathrm{sp}(\mathrm{mix})}\right) / c\right]_{c \rightarrow 0}$ obtained from Castift and Hewtt equation indicating the possible miscibility of the blends. The shift in the respective IR bands further confirms the occurrance of interaction between the polymer phases of the blends. From the thermogravimetric results it was concluded that the improved thermal stability of blends arises from the interaction of degrading PS with the residue of PVAC deacetylation.

\section{REFERENCES}

1. D. R. Paul and S. Newman, "Polymer Blends," Academic Press, New York, 1978.

2. B. Bohmer and S. Florian, Eur. Polym. J., 6, 471 (1970).

3. D. Feldman and M. Rusy, Eur. Polym. J., 6, 627 (1970).

4. I. Inamura and Y. Jinbo, Polym. J., 23, 1143 (1991).

5. W. R. Krigbaum and F. T. Wall, J. Polym. Sci., 5, 505 (1950).

6. E. H. Castift and W. A. Hewtt, J. Appl. Polym. Sci., 6, 962, 530 (1962).

7. M. L. Huggins, J. Am. Chem. Soc., 64, 2716(1942).

8. F. J. Lu, E. Benedetti, and S. L. Hsu, Macromolecules, 16, 1525 (1983). 